\title{
Configuration Space Visualization
}

\author{
Dror Atariah \\ Institut für Informatik \\ Freie Universität Berlin \\ dror.atariah@fu-berlin.de
}

\author{
Günter Rote \\ Institut für Informatik \\ Freie Universität Berlin \\ rote@inf.fu-berlin.de
}

\begin{abstract}
Based on a simple parameterization of contact surfaces, we visualize both the workspace and the corresponding configuration space of a planar polygonal robot that moves amid planar polygonal obstacles.
\end{abstract}

\section{Categories and Subject Descriptors}

G.0 [Mathematics of Computing]: General

\section{General Terms}

Algorithms, Experimentation, Verification

\section{Keywords}

Configuration Space, Workspace, Motion

\section{INTRODUCTION}

Our video has two goals; the first, to visualize the configuration space of a planar polygonal robot moving amid polygonal obstacles, and its correspondence with the actual workspace. The second one is to introduce an explicit parameterization of contact surfaces in the configuration space. These two goals are, as a matter of fact, strongly related, as the actual visualization of the configuration space became feasible by applying the mentioned parameterization.

We consider a planar convex polygonal robot which is free to translate and rotate amid scattered convex polygonal obstacles. Given these three degrees of freedom, the configuration space is three-dimensional, and can be viewed either as $\mathbb{R}^{2} \times \mathbb{R}$ or as $\mathbb{R}^{2} \times S^{1}$. We use the latter representation, and briefly present the former one. We mention the notions of free and forbidden parts of the configuration space, contact surfaces and other related issues. Further background and rigorous discussions can be found in classical textbooks like 2 .

The fundamental motion that we study is a rotation of the robot about one of its boundary points. This motion is represented as a helix in the configuration space, and thus can be easily parameterized. Based on the parameterization of the helices, we easily parameterize the contact object (i.e., surfaces patches or curves) corresponding to the various contact types.

Copyright is held by the author/owner(s).

SCG'12, June 17-20, 2012, Chapel Hill, North Carolina, USA.

ACM 978-1-4503-1299-8/12/06.

\section{Related Work.}

A simple way to get an impression of the three-dimensional configuration space is by taking horizontal slices. This correspondes to keeping the rotation angle fixed and allowing the robot only to translate. The resulting two-dimensional slice of the configuration space is polygonal and can be obtained using Minkowski sums. The sequence of slices can be shown in discrete steps (see for example [1, Appendix F]) or animated on a computer. Our goal is more ambitious: we want to show the obstacles in the configuration space directly and exactly in high quality.

A similar vide ${ }^{1}$ is based on the dissertation of Trac 4 . His video deals with smooth objects, and therefore does not consider notions like the vertex-edge and edge-vertex contacts. In our video, we consider a simpler setting, but we aim for a deeper insight into the configuration space. Another, minor, difference is that we mainly consider the rotation parameter as the bounded interval $[0,2 \pi]$, whereas Trac shows the infinite periodic configuration space.

Finally, another visualization of the configuration space available is the Snake scenarid ${ }^{2}$ which is part of the Master's thesis of Salzman 3]. Here the video shows an instance of a configuration space with a narrow passages, but does not highlight the structure of the various patches of which the contact surfaces are built nor does it visualize any kind of motion.

\section{HELIX IN CONFIGURATION SPACE}

Given a convex polygonal robot, a boundary point $a$ of the robot and some fixed point $P$ in the workspace, we can consider the rotation of the robot about $P$ such that $a$ is fixed to $P$. In this case, the robot's reference point $R_{0}$ traverses, in the workspace, a circle of radius $\left\|R_{0}-a\right\|$ around $P$. This circle lifts, in the configuration space, to a helix. From the parameterization of this helix, we can obtain the parameterization of a vertex-edge contact surface by letting $P$ slide along an edge of an obstacle. Similarly, by choosing a varying boundary point $a$ on one of the robot's edges and setting $P$ to be an obstacle vertex, a parameterization of an edge-vertex contact surface can be found. Thus, the parameterization of these helices is the building block of the various contact surfaces.

${ }_{1}^{1}$ htp://sites.google.com/site/justinscsstuff/ configuration-space-visualization

${ }^{2}$ http://acg.cs.tau.ac.il/projects/mms/videos/ configuration\%20space.wmv 


\section{OVERVIEW OF THE VIDEO}

Along the video, the workspace is always on the right and the configuration space on the left of the frame. In the workspace, the robot is plotted in green, and the obstacles in red. The robot's reference point is marked as a black dot. In the configuration space, the robot is represented as an orange ball, which we call the configuration point; it carries a fixed coordinate frame, where the white arrows point in the $(x, y)$ directions and the purple one points in the vertical (i.e., angular) direction. In the configuration space, blue helices and yellow straight lines correspond to vertex-vertex and edge-edge contacts respectively. Two-dimensional contacts, i.e., vertex-edge and edge-vertex, are plotted in shades of red and green respectively. In order to keep the scenes as clear and understandable as possible, the configuration space, in all but two scenes, is bounded to a gridded bounding box. This bounding box has no mathematical meaning.

\subsection{Contents of the Video}

\section{Introduction.}

In this scene the robot moves in a narrow passage between five obstacles. This scene is very involved and shows all elements of the video which are introduced and discussed in the following sections.

\section{Fundamental Motions.}

We first present a workspace free from obstacles, and we investigate some basic motions of the robot: pure translation, pure rotation and a simple combination of the two.

\section{Helices in the Configuration Space.}

Next, we consider a particular type of motion, namely rotation of the robot, in a workspace still free from obstacles, such that one of its boundary points is fixed to a point in the plane. In this case, the configuration point traverses a helix which is easy to parameterize. Since the contact surfaces are families of helices, we can explicitly construct the contact surfaces using their parameterization.

\section{Contact Surfaces.}

We then introduce an obstacle in the workspace and we study the various possible contact surfaces. In particular, we demonstrate

- a Vertex-Edge contact and the corresponding cylindrical surface patch;

- an Edge-Vertex contact and the corresponding ruled surface patch.

- Finally, we visualize all possible contacts of our triangular robot and an obstacle in detail (see Figure 1). We obtain an overview of this beautiful geometric object, and then we traverse a few particular contacts.

\section{Visualizing a Narrow Passage.}

In the final chapter of the video we come back to the first scene, namely, a narrow passage. Since this is a very complicated scene, we break it up and overlay one obstacle after the other. In this way, the viewer can have a better understanding of how such a passage looks like in the configuration space.

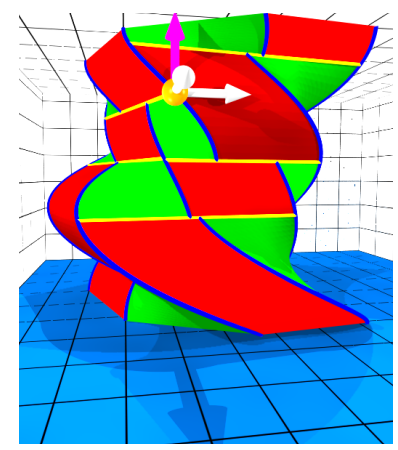

(a) An obstacle represented in the configuration space

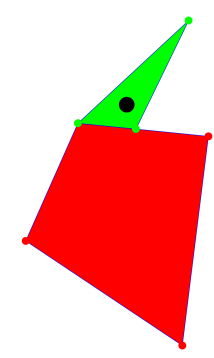

(b) The corresponding configuration of the robot and the obstacle
Figure 1: The two components of a frame

\section{PRODUCTION DETAILS}

This video is the concatenation of a sequence of pairs of frames; each pair consists of an image of the workspace and a corresponding image of the configuration space. In order to describe a continuous motion in the workspace, it is sufficient to provide a continuous path in the configuration space. For each scene in the video, we defined such a path, and extracted, using Mathematica, a dense sample of it. For each point of a sample, we generated, using Mathematica, one frame of the workspace which included the robot in the corresponding pose and the obstacles (if they are present). In addition, from Mathematica we exported the three-dimensional models representing the obstacles into the POV-Ray format. Then, each corresponding image of the configuration space was rendered using POV-Ray.

Finally, the two images of a pair were joined using ImageMagick and the sequence of frames was concatenated into a video using FFmpeg.

\section{ACKNOWLEDGMENT}

This video was made within the project Computational Geometric Learning. The project CG Learning acknowledges the financial support of the Future and Emerging Technologies (FET) program within the Seventh Framework Program for Research of the European Commission, under FET-Open grant number 255827.

\section{REFERENCES}

[1] Howie Choset, Kevin M. Lynch, Seth Hutchinson, George A. Kantor, Wolfram Burgard, Lydia E. Kavraki, and Sebastian Thrun. Principles of Robot Motion: Theory, Algorithms, and Implementations (Intelligent Robotics and Autonomous Agents series). The MIT Press, 52005.

[2] Jean-Claude Latombe. Robot Motion Planning. Kluwer Academic Publishers, 3rd edition, 1993.

[3] Oren Salzman. Motion planning via manifold samples. Master's thesis, Tel Aviv University, 2011.

[4] Steven Cy Trac. Robust Explicit Construction Of 3D Configuration Spaces Using Controlled Linear Perturbation. PhD thesis, University Of Miami, December 2008. 